\title{
Test and Optimization of ABC-System
}

\author{
_Example of HZ Pharmaceutical Company
}

\author{
Feng Zhu \\ School of Accounting, Hangzhou \\ Dianzi University \\ Hangzhou Dianzi University \\ Hangzhou, China
}

\author{
Meiping Lin \\ School of Accounting, Hangzhou \\ Dianzi University \\ Hangzhou Dianzi University \\ Hangzhou, China
}

\author{
Pingxin Wang \\ School of Accounting, Hangzhou \\ Dianzi University \\ Hangzhou Dianzi University \\ Hangzhou, China
}

\begin{abstract}
The debate on activity-based costing (ABC) method between researchers focuses on whether the cost driver selection is scientific and how to prove that $\mathrm{ABC}$ method is superior to the traditional cost accounting method. In this paper, regression model is adopted to test cost drivers' correlation based on historical data from HZ-company that has implemented ABCsystem for 3 years. Further optimization of adjustment quotient to the favorable direction is carried out according to the test results with the purpose of heightening cost drivers' correlation. The test and optimization could verify the scientificity of ABCsystem design and lay a solid foundation for the follow-up activity-based cost management system.
\end{abstract}

\section{Keywords-cost driver; correlation test; optimization}

\section{LITERATURE REVIEW AND COMMENT}

Activity-based costing has become a well-known method for more accurately assigning overhead costs to cost objects, such as products, special orders, and customers, than traditional cost systems (Cooper and Kaplan, 1988).Cost driver is one of the core concepts in ABC. The key to the successful implementation of $\mathrm{ABC}$ is how to select and optimize cost drivers scientifically.

An excessive number of cost drivers have brought complex information cost according to the application of ABC in foreign countries. Cost driver optimization is proposed in this context. It is a combination theory of cost drivers which simplify ABC system as less accuracy loss as possible in accordance with cost-benefit trade-off model in the stage of ABC design. Babad and Balachandran (1993) formulated combination model of two cost drivers; Pingxin Wang etc. (1999) put forward combination model of multiple cost drivers grounded on the former' research and approximation theory of combined cost drivers; Carsten (2001) developed theory of Babad and Balachandran and proposed a model of replacing a cost driver by a combination of the selected cost drivers by linear programming.

Heretofore, the literature and writings about cost driver optimization have focused on cost driver combinations whose aim is to decrease the number of cost drivers in ABC-system and then reduce the complexity of ABC-system together with implementation cost. These studies only combine cost drivers

This paper is the follow-up research achievement of the project " study of Timely Activity-Based Costing” (70172010) supported by the National Natural Science Foundation of China. It's also supported by Zhejiang Provincial “Accounting Engineering Experimental Teaching Demonstration Center” [Zhejiang Educational Office of Higher Education (2010) No.183] and the project "Study of Activity-Based Costing System Optimization" (Y201224759) of Zhejiang Provincial Scientific Research program of the Education Department of China. in the stage of $\mathrm{ABC}$ design and optimize cost drivers in quantitative terms. However, there has not been reported that what is the accuracy grade of the selected cost drivers. Because of different enterprise environment between domestic and foreign countries, system developers in domestic can obtain data related to cost drivers and make optimization of ABCsystem come true. This article will start with testing the cost drivers' correlation to verify the scientificity of ABC design based on historical data from HZ-company that has implemented ABC-system for 3 years and design a set of optimization procedures.

\section{Test ,Optimization TheORY ,Program Design}

\section{A. Test Theory}

Considering the feasible obtainment of cost drivers' data and implementation cost, a sub-optimal driver is frequently selected by empirical method in practice. The empirical method has a certain degree of arbitrariness. If the statistical correlation between the selected suboptimal cost driver and activity based cost is poor, clearly it couldn't explain overhead costs incurred. Hence, conclusion about whether the cost driver selection is scientific could be made in accordance with results from correlation test. If the test results show high correlation, the selected cost driver is reasonable; otherwise, the selected cost driver is unreasonable, which couldn't assign overhead costs more accurately and should be optimized.

Regression method is more accurate than other methods (empirical method and measurement method) in correlation test of cost driver and could achieve more relevant cost data (Doupack, 1993). Regression method discussed in this paper refers to simple linear regression equation whose explanatory variable is cost driver and variable being explained is activitybased cost. So, the test model we use is as follows:

$$
C_{i}=\beta_{0}+\beta_{1} Q_{i}+\varepsilon
$$

where is activity based cost, is quantity of cost driver, is No. of activity core. 


\section{B. Optimization Theory}

\section{1) Optimization Principles}

Materiality principle: More attention should be paid to activity cores of high value. Assigning their activity-based cost inappropriately would easily bring great error of products' cost. So, activity cores of high value will get extra attention in correlation test and raise their criteria; otherwise, we could reduce criteria.

Cost-benefit principle: The design for ABC-system of cost drivers selection have abode by the principle of cost-benefit, which means confirming cost drivers within the range of the maximum implementation cost that the company can bear. If the test results show low correlation of existing cost drivers, the reason is likely to the result of considering implementation cost at the expense of accuracy. Therefore, if the cost of data collection and processing associated with cost drivers for improving accuracy is greater than the accuracy benefits derived from these drivers, the optimal choice is still existing cost driver in spite of low correlation.

\section{2) Optimization Criteria}

In this article, synthetic judgment of correlation will be made based on popular statistical value: t-statistic, F-statistic and R-squared. The F-statistic is significance test of equation. The t-statistic is significance test of regression coefficients. If the Prob. value (F-statistic) and the Prob. value (t-statistic) are lower than the confidence level determined in advance, that is to say, the cost driver passes F-test and t-test. The criteria of Rsquared value differs when involves different cost drivers. A suboptimal driver is usually selected in the analysis of cost and benefit in practice. In addition, many activities may be grouped into a representative cost driver, which cannot explain the whole activity based costs. Therefore, the criteria in this article are formulated combined with the sample HZ: the R-squared of high value activity cores or key activity cores should be higher than 0.64 and the R-squared of those trivial activity cores may lower.

\section{3) Optimization Methods}

For those composite cost drivers that fail to pass the correlation test, 0.618-method is applied in stepwise regression: raise adjustment quotient tentatively and then redistribute costs on the basis of new quotient, test the correlation again; turn to the opposite direction and reduce the adjustment quotient if increasing the adjustment quotient downgrades the R-squared value; increase the adjustment quotient step by step if increasing the adjustment quotient upgrades the R-squared value. The trial ends until the adjustment quotient that can make the R-squared value maximize is sought.

For other cost drivers that fail to pass the correlation test, the replacement existing cost drivers by those drivers that can be easily obtained from the system is a second-choice.

\section{Program Design}

The ABC-system optimization starts with testing the cost driver's correlation as shown in Fig. 1.It's not necessary to carry out optimization if the selected cost driver passes the correlation test; otherwise, optimization of cost driver is essential.

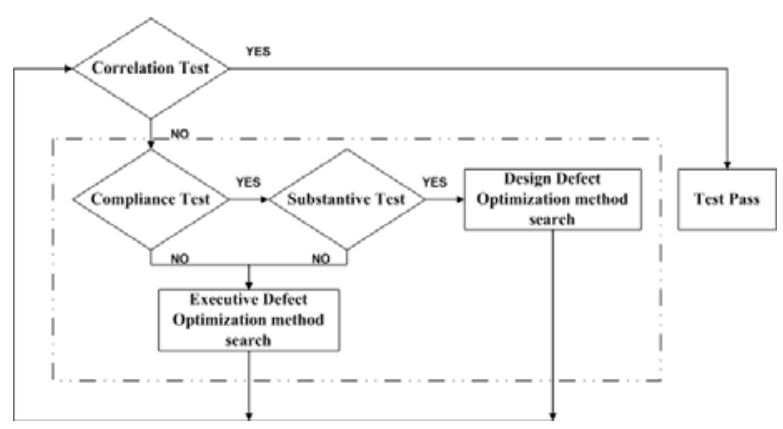

Figure 1. Test and Optimization Program

The 2nd step is compliance test for troubleshooting factors irrelevant with the selected cost driver in the design. The purpose of compliance test is to verify whether the actual running computational logic in ABC-system accord with the design of it. The further step is substantive test if the selected cost driver passes the compliance test. Substantive test refers to censoring the authenticity, accuracy and integrity in aspect of the collection of resources cost, the cost driver of data acquisition and data entry, the computational logic from resources cost to activity core and from activity core to cost objects. Judgment of design defect will be made if the selected cost driver passes both with the addition of failure to pass the correlation test. To this kind of driver, optimization method must be sought to make the final selected cost driver through the correlation test successfully. Judgment of executive defect will be made if the selected cost driver passes the compliance testing, or passes the substantive testing but doesn't pass the compliance testing. For this situation, further efforts to know whether there are difficulties in data collection, storage and processing associated with cost drivers are needed through investigation and analysis. Above all, the cost driver that selected at last can pass the correlation test successfully.

\section{CASE STUDY}

The following article will take a microbial pharmaceutical workshop of HZ Pharmaceutical Company as an example to illustrate test and optimization of ABC-system. $\mathrm{HZ}$ is a Pharmaceutical listed company, which has implemented ABCsystem for 3 years and accumulated a large number of data related to activity-based costing.

\section{A. Correlation Test}

The 1st step is to test the cost driver's correlation according to the above theory. The microbial pharmaceutical consists of 5 activity cores as shown in Table 1.Table 1 also lists 4 products' activity processes. Fig. 2 presents the proportion of workshop's cost. As is shows, the highest proportion is fermentation (73\%), followed by extraction Zone 2 (17\%) and extraction Zone 3 (8\%). 
TABLE I. BRIEF INTRODUCTION TO THE WORKSHOP

\begin{tabular}{|c|c|c|c|}
\hline No. & Appellation of Activity Core & Activity Drivers & Products \\
\hline 1 & Fermentation & Fermentation Tank Cast of Time×Adjustment Quotient & A, B, C, D \\
\hline 2 & Extraction Zone 1 & Batch of Time in Extraction & B, C, D \\
\hline 3 & Extraction Zone 2 & Batch of Time in Refinement & A \\
\hline 4 & Refinement Zone 1 B & Batch of Time in Refinement \\
\hline 5 & Refinement Zone 2 & C, D \\
\hline
\end{tabular}

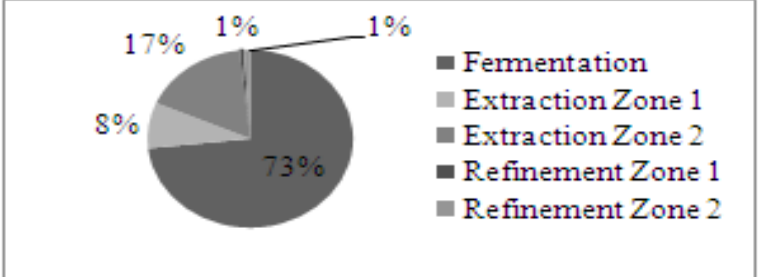

Figure 2. Materiality Analysis of Activity Cores

Table 2 lists the regression results of the original activity drivers, from which we can see that the coefficient of "fermentation tank cast of time $\times$ adjustment quotient" with "activity based cost in fermentation" is 66.2714 and is significantly positive at the 1 per cent level. However, the Rsquared value of that equation is 0.3094 , which means variance of "fermentation tank cast of time $\times$ adjustment quotient" could only explain 30.94 percent of variance of "activity based cost in fermentation”. Conclusion can be drawn that activity driver of fermentation fail to pass the correlation test considering its highest proportion of cost. Table 2 also indicates that the coefficient of "batch of time in extraction" with" activity based cost in extraction” is 1717.213 and is significantly positive at the 1 per cent level, the coefficient of" batch of time in Refinement Zone 1" with "activity based cost in Refinement Zone 1"is 90.9528 and is significantly positive at the 1 per cent level, the coefficient of" batch of time in Refinement Zone 2" with "activity based cost in Refinement Zone 2"is 471.6010 and is significantly positive at the 1 per cent level. In addition, the three equations' R-squared value exceed criterion (0.64). The results above suggest that selections of "batch of time in extraction" and "Batch of Time in Refinement" are scientific and both drivers can assign costs accurately. Conclusion can be drawn that both drivers pass the correlation test.

\section{B. Optimization}

The actual running computational logic of fermentation is in accordance with the original design grounded on a series of investigation activities, including inquiry to director of microbial pharmaceutical workshop and foreman of fermentation, inspection of the statistician's workflow associated with cost of fermentation, review of their working paper related cost. In addition, the collection of resources cost, the cost driver of data acquisition and data entry, the computational logic from resources cost to activity core and from activity core to cost objects is authentic, accurate, integrate. We can conclude that the activity driver of fermentation pass the compliance test and substantive test. However, the adjustment quotient in the original design is only determined by the workshop director according to his experience. It's indispensable to optimize the driver combined with the results of correlation test above.

TABLE II. RESUlts OF ORIGINAL DRIVERS’ CORRELATION TEST

\begin{tabular}{|c|c|c|c|c|}
\hline No. & Variables & Coefficients & R-squared & F-statistic \\
\hline \multirow{2}{*}{1} & INTERCEPT $_{1}$ & $\begin{array}{c}23647766^{* * * *} \\
(5.6499)\end{array}$ & \multirow{2}{*}{0.3094} & \multirow{2}{*}{$9.4066^{* * *}$} \\
\hline & $Q_{1}$ & $\begin{array}{c}66.2714^{* * * *} \\
(3.0670)\end{array}$ & & \\
\hline \multirow{2}{*}{2} & INTERCEPT $T_{2}$ & $\begin{array}{c}26036.57^{* *} \\
(2.2347)\end{array}$ & \multirow{2}{*}{0.8792} & \multirow{2}{*}{$116.4724^{* * * *}$} \\
\hline & $Q_{2}$ & $\begin{array}{c}1717.213^{* * * *} \\
(10.7922)\end{array}$ & & \\
\hline \multirow{2}{*}{4} & INTERCEPT $_{4}$ & $\begin{array}{c}773.2570 \\
(0.2491) \\
\end{array}$ & \multirow{2}{*}{0.7271} & \multirow{2}{*}{$37.3035^{* * * *}$} \\
\hline & $Q_{4}$ & $\begin{array}{c}90.9528^{* * * *} \\
(6.1077)\end{array}$ & & \\
\hline \multirow{2}{*}{5} & INTERCEPT $_{5}$ & $\begin{array}{c}8186.462^{*} \\
(1.9104)\end{array}$ & \multirow{2}{*}{0.7886} & \multirow{2}{*}{$63.4321^{* * * *}$} \\
\hline & $Q_{5}$ & $\begin{array}{c}471.6010^{* * * *} \\
(7.9644)\end{array}$ & & \\
\hline
\end{tabular}


The original design set the other three products' adjustment quotient in the light of product $\mathrm{A}$, whose adjustment quotient is 1. Product B's quotient is 1.2, Product C's quotient is 1.4 and Product D's quotient is 0.8 . We use Product B for example to explain how to carry out optimization of composite cost drivers. Adjustment quotient is raised to $1.9(1.2 \div 0.618)$ and then redistribute overhead costs on the basis of 1.9 , test the correlation again. The results are shown in Table 3 . The coefficient of "fermentation tank cast of time $\times$ adjustment quotient" with "activity based cost in fermentation” is 33.6404 and is significantly positive at the 5 per cent level. The Rsquared value decreases from 0.3094 to 0.2194 . So, the further step is turn to the opposite direction and reduce the adjustment quotient to $0.7(1.2 \times 0.618)$. Table 3 suggests that the $\mathrm{R}$ squared value gets growth up to 0.4516 based on quotient 0.7 and the R-squared value continues rising to 0.4531 based on quotient $0.4(0.7 \times 0.618)$ but decline to 0.3808 based on quotient 0.3 . To sum up, adjustment quotient of Product $B$ should be set in 0.4 rather than 1.2.

TABLE III. RESULTS OF OPTIMIZATION

\begin{tabular}{|c|c|c|c|c|}
\hline Quotient & Variables & Coefficients & R-squared & F-statistic \\
\hline \multirow{2}{*}{1.2} & INTERCEP $T_{1}^{1}$ & $\begin{array}{c}2364776^{* * *} \\
(5.6499)\end{array}$ & \multirow{2}{*}{0.3094} & \multirow{2}{*}{$9.4066^{* * * *}$} \\
\hline & $Q_{1}^{1}$ & $\begin{array}{c}66.2714^{* * * * *} \\
(3.0670)\end{array}$ & & \\
\hline \multirow{2}{*}{1.9} & INTERCEPT $T_{1}^{2}$ & $\begin{array}{c}2689536 \\
(6.8188)\end{array}$ & \multirow{2}{*}{0.2194} & \multirow{2}{*}{$5.9026^{* *}$} \\
\hline & $Q_{1}^{2}$ & $\begin{array}{l}33.6404^{* *} \\
(2.4295) \\
\end{array}$ & & \\
\hline \multirow{2}{*}{0.7} & INTERCEPT $T_{1}^{3}$ & $\begin{array}{l}1908364^{* * * *} \\
(4.5581)\end{array}$ & \multirow{2}{*}{0.4516} & \multirow{2}{*}{$17.2952^{* * * *}$} \\
\hline & $Q_{1}^{3}$ & $\begin{array}{l}135.0656^{* * *+1} \\
(4.1588)\end{array}$ & & \\
\hline \multirow{2}{*}{0.4} & INTERCEPT $T_{1}^{4}$ & $\begin{array}{c}2043425^{* * *} \\
(5.3024) \\
\end{array}$ & \multirow{2}{*}{0.4531} & \multirow{2}{*}{$17.3972^{* * * *}$} \\
\hline & $Q_{1}{ }^{4}$ & $\begin{array}{c}177.7366^{* * * *} \\
(4.1710)\end{array}$ & & \\
\hline \multirow{2}{*}{0.3} & INTERCEPT $T_{1}^{5}$ & $\begin{array}{c}2355001^{* * * *} \\
(6.5274)\end{array}$ & \multirow{2}{*}{0.3808} & \multirow{2}{*}{$12.9140^{* * * *}$} \\
\hline & $Q_{1}^{5}$ & $\begin{array}{c}166.6950^{* * * *} \\
(3.5936)\end{array}$ & & \\
\hline
\end{tabular}

It is unnecessary to go into details of C and D's because of similar optimization method.

\section{CONCLUSION AND OUTLOOK}

This study tests the statistical correlation of 5 cost drivers from HZ's microbial pharmaceutical workshop by simple linear regression model. The driver of fermentation fails to pass the test and is optimized finally according to the test results. The results of this paper not only verify the scientificity of ABC system design, but also lay a solid foundation for the follow-up activity-based cost management system.

There still exists space extended in the following aspects: only simple linear regression model is put into use to test drivers' correlation. Is the multiple linear regression model applicable? How do the researchers apply the model? The key problem that must be addressed in practice is how to formulate multi-level criteria of the R-squared value scientifically.

\section{REFERENCES}

[1] Cooper, R. and Kaplan, R.S., "Measure cost right : make the right decision," Harvard Buisiness Review, vol.66, pp. 96-103, 1955.

[2] M. Babad, V. Balachandran, "Cost driver optimization in activity-based costing,” Accounting Review,pp. 529-551, July 1993.

[3] Pingxin Wang, Fangjun Wang, and Peng Wang, "Study on Homogeneity of cost drivers," The Journal of quantitative \& Technical Economics , vol- 5,1999, pp.44-46.

[4] Carsten Homburg, "A note on optimal cost driver selection in ABC," Management Accounting Research, pp. 199-203, December 2001.

[5] Doupack N, “A Perspective On Cost Drivers,” Accounting Review, pp. 121-126, July1993. 